Die „Just in Time-Funktion“ bestellt den Patienten in die Praxis, wenn er auch wirklich an der Reihe ist. Der Patient kann bei der Terminvereinbarung seinen Standort angeben, das System kalkuliert dann die Zeit und gibt dem Patienten rechtzeitig Bescheid, wann er sich auf den Weg zum Arzt machen soll.

Eine weitere Entlastung für das Praxispersonal ist laut Adendorff der „Wartezimmer-Organizer“. Ein Monitor im Wartezimmer gibt den Patienten Bescheid, in welches Behandlungszimmer sie zu gehen haben. Auch kennt das System den Grund für den Arztbesuch und kann so die Zeit für die Behandlung kalkulieren. „Die Information zu Praxiszimmer, Dauer der Behandlung und ähnliches muss einmalig bei der Installation festgelegt werden, danach arbeitet das System alleine“, erklärt Adendorff.

Das „Sprechzimmermodul“ informiert den Arzt in welchem Zimmer sich der nächste Patient befindet, wer er ist und was der Grund für seinen Besuch ist.
Adendorffs Ziel war, dass mit AJITS ${ }^{\oplus}$ Arzt und MFA wieder mehr Zeit für die Patienten haben. „Auch fallen dadurch Überstunden weg, die Mitarbeiter kommen pünktlich nach Hause, was die $\mathrm{Zu}$ friedenheit im Team steigert." Laut Adendorff spart das System der MFA zwei und dem Arzt eine Stunde Zeit am Tag.

AJITS $^{\circledast}$ funktioniere nicht nur in Einzelpraxen, sondern auch in Gemeinschafts-praxen, MVZ und Kliniken, so der Entwickler. Es können mehrere Ärzte und auch Wartezimmer in das System eingepflegt werden. „Es ist sehr flexibel und mit jedem Praxissystem kompatibel“, sagt Adendorff. Die Kosten für eine Einzelpraxis belaufen sich auf etwa $2.000 €$ für alle fünf Module. Es lassen sich aber auch nach Bedarf einzelne Module kaufen.

Adendorff hat mittlerweile auch schon die nächsten Ideen. Bei seiner Recherche in der Hausarztpraxis ist ihm aufgefallen, wie lange die Abrechnung dauert. „Hier kann man auch noch einiges optimieren", ist er sich sicher. Kerstin Mitternacht
Nationale Leitlinien jetzt auch per App verfügbar

Die "Nationalen Versorgungsleitlinien" (NVL) können sich Ärzte jetzt auch per App auf iPhone oder iPad ansehen. Das geht über die App „VersorgungsLeitlinien" des Ärztlichen Zentrums für Qualität in der Medizin (ÄZQ), die kostenfrei im iTunes-Store (ihttp://itunes.apple.com) heruntergeladen werden kann. Nutzer finden in der App die vollständige Kurzfassung der Versorgungsleitlinien - inklusive aller Handlungsempfehlungen, erläuternden Hintergrundtexten und Übersichtstabellen.

Die App ist auch für das Patientengespräch interessant. Sie enthält spezielle Patienteninformationen, genauer werden kurz und für Patienten verständlich Erkrankungen erklärt und es wird auf weiterführendes Info-Material verwiesen. Bislang sind allerdings nur die NVL zu Asthma, Kreuzschmerz und Herzinsuffizienz verfügbar. Nach und nach sollen aber alle NVL für die App aufbereitet werden, meldet das ÄZQ. Dabei steht das Programm für Nationale Versorgungsleitlinien unter der Trägerschaft von Bundesärztekammer, Kassenärztlicher Bundesvereinigung und der Arbeitsgemeinschaft der Wissenschaftlichen Medizinischen Fachgesellschaften. Das ÄZQ wurde allerdings mit der Umsetzung der Leitlinien beauftragt. reh

\section{medatixx und samedi legen} Online-Kalender auf

Der Arztsoftware-Anbieter medatixx bietet seinen Kunden seit 1. Oktober einen Online-Terminkalender an. Der webbasierte Kalender "ixx.time powered by samedi" ist ein gemeinschaftliches Projekt mit dem Web-Software-Anbieter samedi, der bereits seit Jahren ein OnlineTerminbuchungssystem für Praxen bereitstellt. Über den Online-Kalender kann laut medatixx nicht nur die TerminKommunikation mit Patienten, sondern auch mit anderen Praxen und Kliniken laufen.

Zum Start des Online-Kalenders bietet medatixx Ärzten eine besondere Aktion: Um die Wartezeit auf den neuen Kalender zu verkürzen, würden die ersten 500 Interessenten, die per E-Mail an info@medatixx.de Informationen anfordern oder via Kommentar auf facebook. de/medatixx oder per \#ixxtime-Twitternachricht @medatixx ihr Interesse signalisieren, gratis einen ixx.time-Lichtwecker erhalten. 